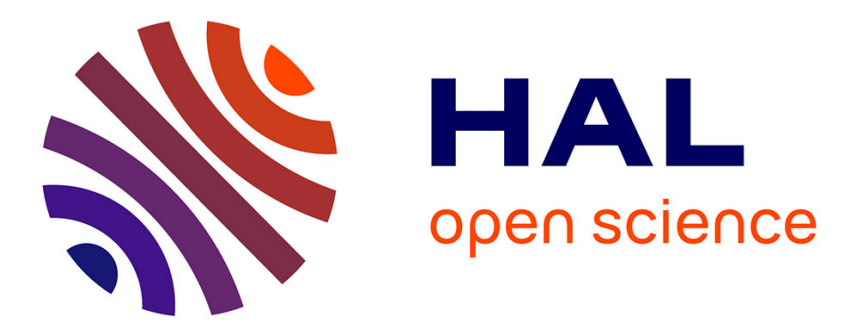

\title{
Shear stress statistics and wall similarity analysis in turbulent boundary layers using a high-resolution 3-D ADVP
}

David Hurther, Ulrich Lemmin

\section{- To cite this version:}

David Hurther, Ulrich Lemmin. Shear stress statistics and wall similarity analysis in turbulent boundary layers using a high-resolution 3-D ADVP. IEEE Journal of Oceanic Engineering, 2001, 25 (4), pp.446-457. 10.1109/48.895352 . hal-00230300

\section{HAL Id: hal-00230300 \\ https://hal.science/hal-00230300}

Submitted on 27 Mar 2020

HAL is a multi-disciplinary open access archive for the deposit and dissemination of scientific research documents, whether they are published or not. The documents may come from teaching and research institutions in France or abroad, or from public or private research centers.
L'archive ouverte pluridisciplinaire HAL, est destinée au dépôt et à la diffusion de documents scientifiques de niveau recherche, publiés ou non, émanant des établissements d'enseignement et de recherche français ou étrangers, des laboratoires publics ou privés. 


\title{
Shear Stress Statistics and Wall Similarity Analysis in Turbulent Boundary Layers Using a High-Resolution 3-D ADVP
}

\author{
David Hurther and Ulrich Lemmin
}

\begin{abstract}
Three-dimensional (3-D) quasi-instantaneous acoustic Doppler velocity profiles at the center of uniform, turbulent open-channel flow over smooth and rough beds have been analyzed for the dynamics of coherent structures. The qualitative aspects of simultaneously measured Eulerian velocity and shear stress signatures identify coherent structures in the water column. A cumulant discard method is applied to describe the statistical properties of the covariance terms $u^{\prime} w^{\prime}$ along the mean flow and $v^{\prime} w^{\prime}$ across the mean flow relative to their time means. Conditional statistics and conditional sampling are used to compare the theoretical and experimental relative covariance contributions from the four quadrants in the longitudinal and transverse planes. The results in the $\left(u^{\prime}, w^{\prime}\right)$ plane show the dominance of ejections (quadrant 2; $u^{\prime}<0, w^{\prime}>0$ ) and sweeps (quadrant $\left.4 ; u^{\prime}>0, w^{\prime}<0\right)$. In contrast, the distribution of fractional $v^{\prime} w^{\prime}$ events in the transverse plane is quasi-uniform over the four quadrants. Based on these experimentally determined statistical properties of the covariance terms in different flow conditions, a simplified form of the vertical turbulent energy flux in the intermediate flow region is given and the concept of wall similarity in turbulent boundary layers is validated. Since the validity of the wall similarity concept over a wide range of bed roughness has been shown, it is proposed to determine the mean bed friction velocity from the evaluation of the vertical turbulent energy flux.
\end{abstract}

Index Terms-Coherent structures, conditional sampling, Doppler measurement, random media, turbulent bursts.

\section{INTRODUCTION}

$\mathbf{O}$ VER the past decade, Doppler sonars have become powerful instruments for the measurement of oceanic current profiles. The type of instrument most often used applies the "noncoherent" technique [18]. More recently, oceanic research has focused increasingly upon boundary layer studies in nearshore and coastal environments and in benthic boundary layers. In these applications, the depth range of interest is relatively short $(\mathrm{O}(1 \mathrm{~m}))$ and, due to limitations inherent in "noncoherent" sonars, such instruments can no longer be used efficiently. Point measurement techniques have been applied in this environment. However, acoustic profiling using the "pulse-topulse" coherent technique [10], [11] offers an attractive alternative because it is possible to cover the total depth of the boundary layer, and the technique is nonintrusive.

The authors are with the Laboratoire de Recherches Hydrauliques, Ecole Polytechnique Federale Lausanne, CH-1015 Lausanne, Switzerland.
Boundary layer flows are often characterized by strong vertical gradients. The pulse-to-pulse coherent technique can provide the high spatial $(\mathrm{O}(5 \mathrm{~mm}))$ and temporal $(\mathrm{O}(15 \mathrm{~Hz}))$ resolution as well as the high velocity resolution $\left(\mathrm{O}\left(3 \mathrm{mms}^{-1}\right)\right)$ required to study the details of boundary layer flow. By changing the acoustic frequency and the system geometry, the system can be adapted to the boundary layer dimensions encountered in the field.

The presence of high spatial and temporal variability in the boundary layer flow field has been documented in the literature. The streamwise velocity field in the near-wall region of a boundary layer is known to be organized in alternating streaks of high and low velocity which are persistent in time. Related to this structure are intermittent, quasi-periodic events, consisting of outward ejections of low-speed fluid from the bed and inrushes of high-speed fluid toward the bed, called coherent structures. From studies of wall-bound turbulent shear flows, these organized motions (i.e., the bursting phenomena) are identified as a primary turbulence mechanism for the production of Reynolds stress and turbulence energy in the near-bed region [8], [15]. Little is known so far about the dynamics of coherent structures in the cross-stream plane.

Profiling the whole depth of the boundary layer in a single sweep is an ideal technique to study the dynamics of these coherent structures. They have been made evident using 2-D [21] and 3-D [5] acoustic Doppler velocity profilers in various flow conditions. Recently, we have also shown their relation to sediment resuspension and transport using an acoustic flux profiler [23], [7].

The cumulant discard method has been used by several authors, all of whom used point measurement techniques, in order to predict quantitatively the covariance $u^{\prime} w^{\prime}$ term statistics in the streamwise direction for different wall roughness conditions in zero pressure gradient boundary layers. Antonia and Atkinson [1] estimated the skewness and flatness factors of the shear stress fluctuations by a fourth-order approach and expressed the probability density distribution of the normalized $u^{\prime} w^{\prime}$ term from a Gram-Charlier type joint probability density function of $u^{\prime}$ and $w^{\prime}$. In the inner region of the flow, they found good agreement between results of the cumulant discard method and the corresponding experimental factors. Only a qualitatively similar tendency was observed in the outer region where the flow was found to be more intermittent.

Nakagawa and Nezu [16] developed the equations of the probability density for the relative covariance term $u^{\prime} w^{\prime}$ conditionally sampled over the four quadrants of the $\left(u^{\prime}, w^{\prime}\right)$ 
plane. Events with quadrant $1\left(u^{\prime}>0, w^{\prime}>0\right)$, quadrant 2 $\left(u^{\prime}<0, w^{\prime}>0\right)$, quadrant $3\left(u^{\prime}<0, w^{\prime}<0\right)$ and quadrant $4\left(u^{\prime}>0, w^{\prime}<0\right)$ orientation are respectively identified as outward interaction, ejection, inward interaction, and sweep events. They showed that a third-order Gram-Charlier distribution is sufficient to quantify the quadrant time fractions and quadrant covariance fractions in the wall region $(z / h<0.1$, where $h$ is the water depth) and in the equilibrium region $(0.15<z / h<0.6)$ of open-channel flow.

Raupach [19] applied a third-order Gram-Charlier type distribution to quantify the covariance statistic parameters in rough-wall and smooth-wall turbulent wind tunnel boundary layers. These three studies agree on the effect of roughness on the dynamics of shear stress events $u^{\prime} w^{\prime}$ : in the vicinity of the wall, it is characterized by an increase of sweep event contribution, both with roughness and with proximity to the wall.

This paper reports on an experimental investigation of the conditionally sampled covariance events $u^{\prime} w^{\prime}$ in the streamwise direction and $v^{\prime} w^{\prime}$ in the across stream plane. In order to evaluate the dynamics under controlled conditions, experiments were carried out in turbulent open-channel flow over smooth and rough beds using a 3-D acoustic Doppler velocity profiler. An interpretation of the Eulerian signatures of the covariance and 3-D velocity fields over the whole depth will be presented.

To validate the effect of roughness on covariance dynamics, experimental results of fractional contribution to the corresponding shear from each quadrant of both covariance terms will be compared to results from a cumulant discard Gram-Charlier distribution, applied to both $u^{\prime} w^{\prime}$ and $v^{\prime} w^{\prime}$ terms.

From the observed linear relation of the third-order moments in the equilibrium flow region of the boundary layer, a simplified form of the vertical turbulent energy flux is validated. Since a constant value of the normalized turbulent kinetic energy flux is found in the flow region $(0.25<z / h<0.75)$, a method for the determination of the bed friction velocity is proposed which has certain advantages over commonly used methods.

\section{ACOUSTIC DOPPLER Velocity PROFILER}

A pulse-to-pulse coherent acoustic Doppler velocity profiler (ADVP) is used to evaluate the three velocity components simultaneously over the entire investigated water depth. The system consists of a central narrow beam emitter and up to four wide-angle receiver transducers placed symmetrically around the center. The transducers are arranged in two perpendicular planes, each of which allows one to resolve profiles of one horizontal and the vertical velocity component. The redundancy of the vertical component provides for a control of the quality of the geometrical alignment of the transducers. Since sound is emitted only from the central transducer, all velocity components are evaluated from phase information coming from the same scattering volume. Unlike commercially available profilers, the ADVP measures velocities along a single straight line of consecutive scattering volumes. This is the only way in which turbulence information and particularly a correlation between velocity components can correctly be evaluated without any specific requirements for the flow conditions. Quasi-instantaneous velocity components are estimated using the pulse-pair algorithm. The system is described in more detail in [11], [21], and [12].

A combination of 1) a hardware method and 2) a software method is used here to reduce noise contributions in the turbulence measurements with the 3-D ADVP. A brief explanation of the two methods is given.

1) An ultrasonic constant beamwidth transducer system is used which is capable of generating an extended focal zone by electronically focusing the beam over the desired water depth range [5], [12]. Beam directivity measurements show that the higher beam directivity and the reduction in side-lobe level lead to an increase of the signal-to-noise ratio (SNR) by up to $15 \mathrm{~dB}$ compared to a plane disc transducer. It also leads to significant reduction in spectral broadening effects, which in the case of plane disc transducers reduce the velocity resolution and interfere with correct data interpretation [11].

2) A direct Doppler signal treatment method is applied to the data [6]. The technique does not rely on any hypothesis concerning flow characteristics. No knowledge about supplementary device-dependent parameters such as the sample volume size or the acoustic beam opening angle are needed. It is based on a noise spectrum reconstruction from cross-spectra evaluations of two simultaneous and independent measurements of the vertical velocity over the whole water depth. Correcting for the system geometry, the noise spectra and the noise variances are calculated and removed from the three fluctuating velocity components. The corrected turbulence spectra show a $-5 / 3$ slope over the whole inertial subrange delimited by the frequency band of the device.

The corrected profiles of turbulence intensities, turbulent kinetic energy, shear stress, and turbulent energy balance equation terms such as production, transport, and dissipation are in good agreement with different semitheoretical formula and other measurements from the literature.

The combination of this correction method with the use of the constant beamwidth emitter allows turbulence measurements with a relative error under $10 \%$ in the intermediate and free surface flow regions $(0.15<z / h<1)$ of the boundary layer. As indicated in [6], deviations of the corrected mean turbulence measurements from semitheoretical laws are observed in the wall region. Supplementary studies are needed to evaluate quantitatively the limits of sonar measurements in that flow domain. While we allocate the remaining variations to effects of the spatial averaging due to the strong profile gradients in this layer, effects of bottom roughness cannot be excluded either.

\section{EXPERIMENTAL SETUP}

Experiments were carried out in two laboratory open-channels under uniform flow conditions: the first one $(29 \mathrm{~m}$ long, $2.45 \mathrm{~m}$ wide, $75 \mathrm{~cm}$ deep) has a rough bed and the second one (43 m long, $2 \mathrm{~m}$ wide, $1 \mathrm{~m}$ deep) has a smooth bed. For experiments over rough beds (experiments B, C, and D in Table 
TABLE I

HYDRAULIC PARAMETERS FOR EXPERIMENTS

\begin{tabular}{ccccccccccccc}
\hline Exp. & $\begin{array}{c}\mathrm{Q} \\
\left(\mathrm{m}^{3} / \mathrm{s}\right)\end{array}$ & $\begin{array}{c}\mathrm{h} \\
(\mathrm{cm})\end{array}$ & $\begin{array}{c}\mathrm{U} \\
(\mathrm{cm} / \mathrm{s})\end{array}$ & $\begin{array}{c}\mathrm{u}_{*} \\
(\mathrm{~cm} / \mathrm{s})\end{array}$ & $\begin{array}{c}\mathrm{u}_{\mathrm{k}, \mathrm{s}} \\
(\mathrm{cm} / \mathrm{s})\end{array}$ & $\begin{array}{c}\mathrm{u}_{*, j o g} \\
(\mathrm{~cm} / \mathrm{s})\end{array}$ & $\begin{array}{c}\mathrm{S} \\
\left(\times 10^{4}\right)\end{array}$ & $\begin{array}{c}\mathrm{Re}_{\mathrm{h}} \\
\left(\times 10^{3}\right)\end{array}$ & $\mathrm{Fr}_{\mathrm{h}}$ & $\mathrm{B} / \mathrm{h}$ & $k_{s}^{+}$ & $\Pi$ \\
\hline $\mathrm{A}$ & 0.115 & 19.1 & 32 & 2.1 & 2.3 & 2.3 & 2.8 & 61 & 0.23 & 10.5 & $\approx 0$ & 0.4 \\
$\mathrm{~B}$ & 0.069 & 10.6 & 25.5 & 2.0 & 2.3 & 2.0 & 5 & 27 & 0.25 & 23 & 34 & 0.1 \\
$\mathrm{C}$ & 0.122 & 16 & 31 & 2.5 & 2.8 & 2.7 & 5 & 49.6 & 0.25 & 15.3 & 45 & 0.1 \\
$\mathrm{D}$ & 0.200 & 17.3 & 47.5 & 2.9 & 2.9 & 3.0 & 5 & 82.2 & 0.36 & 14.2 & 50 & 0.3 \\
\hline \hline
\end{tabular}

I), a sand bed with a mean grain size of $d_{50}=1.7 \mathrm{~mm}$ (estimated at $50 \%$ of the granulometric curve) was used. The measurement sections are placed 13 and $16 \mathrm{~m}$ downstream from the entrances for rough and smooth bed conditions, respectively, where the turbulent flow is well developed. All velocity data presented here were taken in the center of the channels. The hydraulic parameters are given in Table I. These indicate subcritical highly turbulent flows for all experiments with varying Reynolds, Froude numbers, and bed friction velocities. The uncertainties on discharges, water depths, and discharge velocities estimations shown in Table I are less than 5\% and less than 10\% for the bed shear-velocity estimations.

The variables $u_{*}, u_{*, S}$ and $u_{*} \log$ represent the friction velocities obtained from linear extrapolation of the mean Reynolds stress at the wall, from the energy line slope formula for uniform flow, and from the log law, respectively. The relative errors (relative to $u_{*}, \log$ ) are less than $10 \%$ which shows that conditions of uniform flow are satisfied. The standard roughnesses $k_{S}^{+}$are normalized with the corresponding viscous lengths and are smaller than 70 , for all experiments, corresponding to incompletely rough beds found in oceanic boundary layers. A Manning-Strickler formula is used to estimate the physical grain size from the uniform flow conditions in the cases of rough beds (experiments B-D in Table I). We obtain values between 3 and $5 \mathrm{~mm}$ for the mean particle diameter $d_{50}$. The difference between the calculated values and the true value $(1.7 \mathrm{~mm})$ indicates the difficulty to determine realistic results from profile extrapolations for rough bed bottoms where the position of the profile origin is poorly defined. The $\Pi$ factor of Coles wake function (Table I) is extracted from the velocity defect law [2] with $\kappa$ equal to 0.4 and varies between 0.1 and 0.4 .

The experiments were conducted under clear water conditions where particles do not contribute significantly to backscattering. Shen and Lemmin [22] identified the ultrasonic targets as being concentration microstructures originating from clusters of fine air bubbles. Since these structures have a very small size, they are able to follow the turbulent motion without inertial lag over the investigated frequency band. All measurements presented here are extracted from data sets acquired over 600-s intervals. The spatial and temporal resolutions are dependent on the settings of the instrument and are equal to $\cong 3 \mathrm{~mm}$ and 0.024 $\mathrm{s}$, respectively, in the present case.

\section{ObServations of COMMON STRUCTURAL Flow FEATURES OVER SMOOTH AND ROUGH BEDS}

Eulerian signatures are limited for the identification of the different types of coherent structures passing through a fixed measurement volume because they have a high spatio-temporal evolution [20]. In order to compare our data qualitatively to simultaneous velocity measurements over an entire cross section presented in Grass et al. [4], a Lagrangian flow field was computed by applying Taylor's hypothesis for the mean flow direction. As in Grass et al. [4], a convection velocity, $U_{d}$, equal to the depth-averaged mean velocity was chosen in order to compare the measurements. Fig. 1 shows four sequences (of our data) where the covariance and fluctuating velocity patterns are superimposed. Fig. 1(a) presents the fluctuating 2-D velocity vectors $\mathbf{V}\left(u-U_{d}, w^{\prime}\right)$ and covariance iso-contourlines in the longitudinal flow plane in rough (experiment A) and in smooth [experiment D, Fig. 1(b)] bed conditions. Fig. 1(c) and (d) give the same information in the transverse flow plane for rough (experiment D) and smooth (experiment A) bed conditions, respectively.

The diagrams in Fig. 1 show flow structures similar to those observed by Grass et al. [4]. Flow patterns such as vortex cross sections, internal shear layers, ejections, sweeps, upwellings, and downwellings over a smooth bed are identical to those over a rough bed. For example, internal high-shear layers are observed in Fig. 1(a) at $x=0.8 \mathrm{~m}, z / h=0.25$, corresponding to a sweep event (quadrant 4 direction) and in Fig. 1(b) at $x=1.1$ $\mathrm{m}, z / h=0.125$, corresponding to an ejection event (quadrant 2 direction). Vortex cross sections can be seen in the longitudinal [Fig. 1(a) at $x=0.36 \mathrm{~m}, z / h=0.42$ and Fig. 1(b) at $x=0.1 \mathrm{~m}, z / h=0.45$ ] and transverse [Fig. 1(c) at $x=0.68$ $\mathrm{m}, z / h=0.375$ and Fig. 1 (d) at $x=1.23 \mathrm{~m}, z / h=0.8$ ] flow fields indicating the three-dimensionality of the vortical structures. Vortex cross sections associated with an underlying high shear layer such as an ejection event can be observed at $x=1.49$ $\mathrm{m}, z / h=0.25$ in Fig. 1(a).

Numerical boundary layer simulation results of Robinson et al. [20] show similar structures where the vortex core is identified as the head of a horseshoe vortex located above an ejection event. In the free surface flow region, upwellings and downwellings can be seen at several locations in the flow fields [in Fig. 1(a) at $x=1.06 \mathrm{~m}, z / h=0.85$ and in Fig. (b) at $x=1.32 \mathrm{~m}, z / h=0.88]$. As shown by Kumar et al. [9] by instantaneous Lagrangian flow visualization, these time-persistent free-surface structures (especially upwellings) were observed to generate one or more pairs of spiral eddies at the water surface with opposite normal vorticities. They suggested that this sequence of events might be typical for the generation process of water surface structures such as boils in turbulent free surface flows.

Previous [21], [23] and present observations of different kinds of coherent structures give support to the existence of flow pat- 


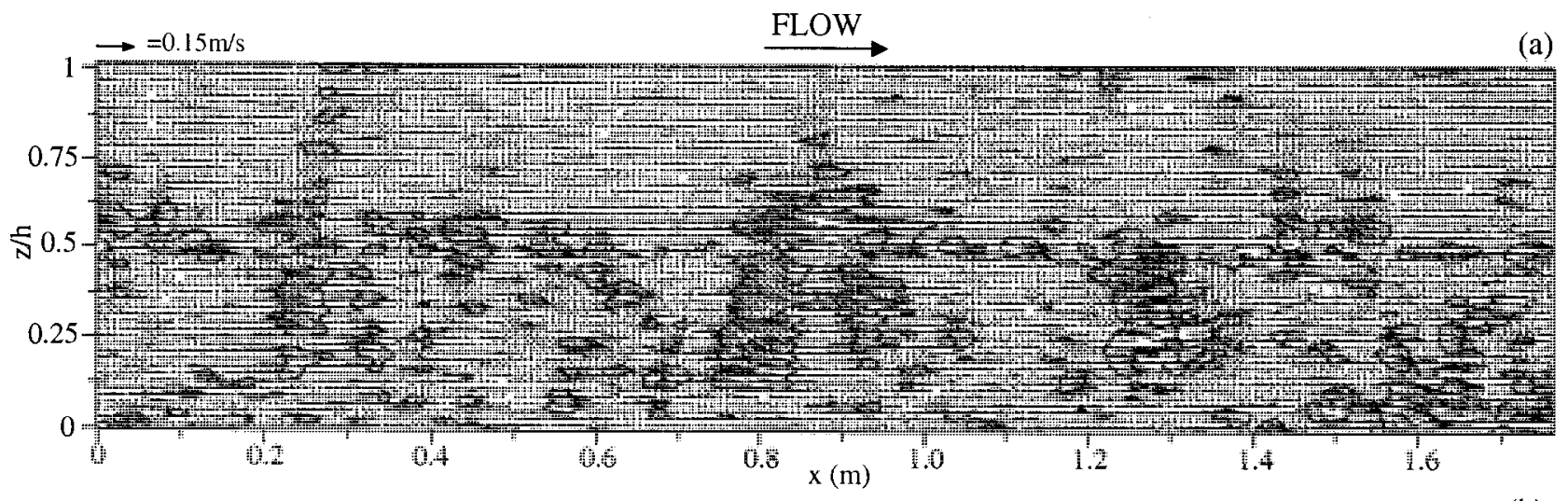

(a)
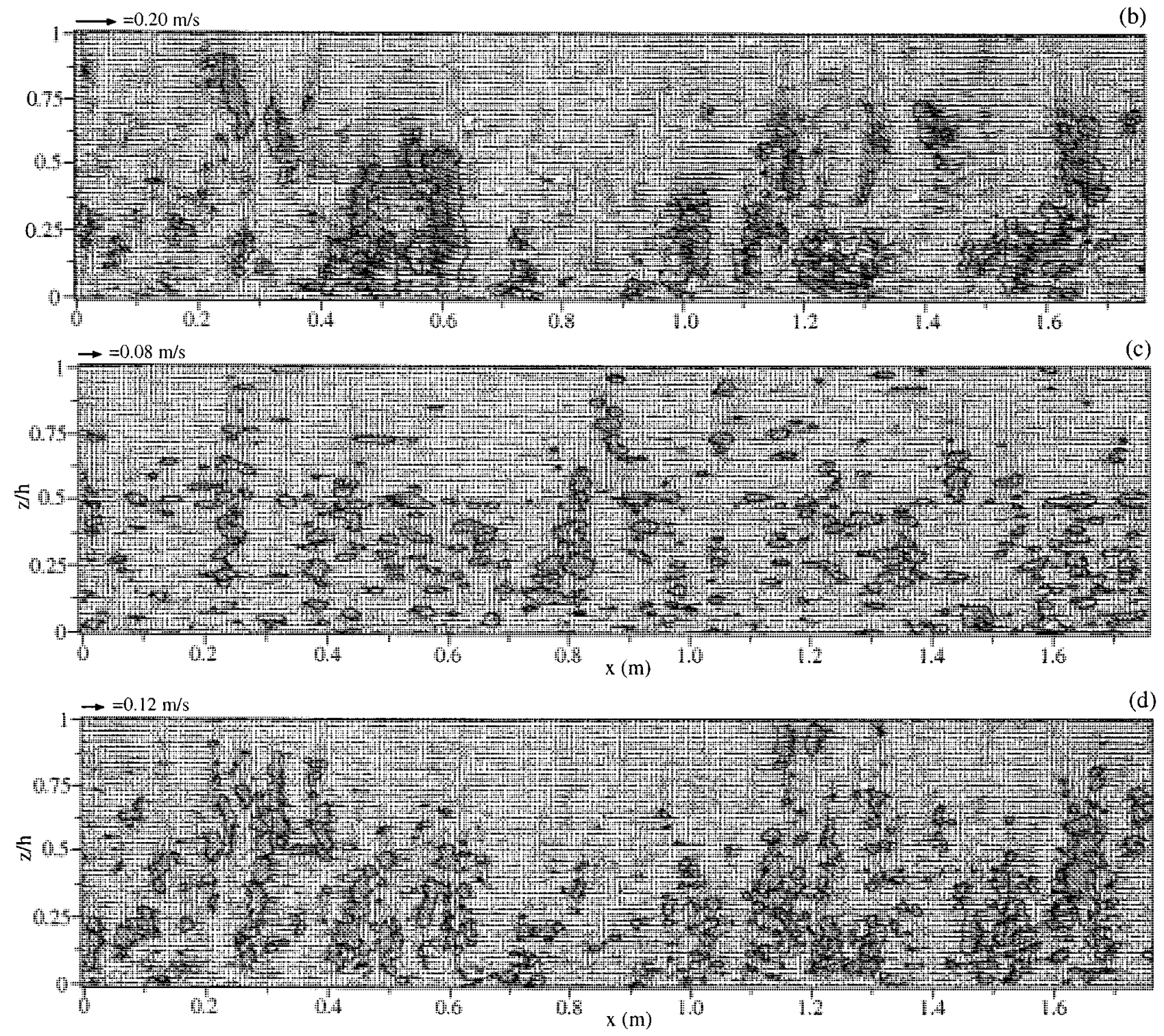

Fig. 1. Iso-contours of high shear patterns and fluctuating velocity field at centerline of the channel for (a) the $\mathbf{V}^{\prime}\left(u^{\prime}, w^{\prime}\right)$ velocity vector (in Exp. A), (b) the $\mathbf{V}^{\prime}\left(u^{\prime}, w^{\prime}\right)$ velocity vector (in Exp. D), (c) the $\mathbf{V}^{\prime}\left(v^{\prime}, w^{\prime}\right)$ velocity vector (in Exp. A), and (d) the $\mathbf{V}^{\prime}\left(v^{\prime}, w^{\prime}\right)$ velocity vector (in Exp. D).

terns common to both smooth and rough beds. Furthermore, the matching of these flow patterns with the patterns shown in [4] confirms the presence of horseshoe vortical structures, although they could not be visualized spatially with our measurement technique. In the longitudinal flow plane, ejection and sweep events seem to be predominant. No dominant patterns can be distinguished in the transverse shear stress structures [Fig. 1(c) and (d)]. This observation will be confirmed below by quantifying the 3-D statistical properties of the longitudinal and transverse relative covariance terms. 


\section{Conditional Statistics of Relative Covariances}

\section{A. Theoretical Aspects}

In order to apply the cumulant discard method, we define the following variables: $u^{\prime}, v^{\prime}, w^{\prime}$ are the zero mean fluctuating longitudinal, transverse, and vertical velocity components, respectively. $\widehat{u}, \widehat{v}, \widehat{w}$ are equal to $u^{\prime} / \sqrt{\overline{u^{\prime 2}}}$, $v^{\prime} / \sqrt{\overline{v^{\prime 2}}}, w^{\prime} / \sqrt{\overline{w^{\prime 2}}}$. We shall quantify the contributions from the different planes of the relative shear stress for the covariance terms $\varepsilon_{1}=u^{\prime} w^{\prime} \overline{u^{\prime} w^{\prime}}$ and $\varepsilon_{2}=v^{\prime} w^{\prime} / \overline{v^{\prime} w^{\prime}}$. Fig. 2 shows the orientation of the quadrants in the longitudinal and transverse planes. Two characteristic functions $\phi_{1}(\widehat{u}, \widehat{w})$ and $\phi_{2}(\widehat{v}, \widehat{w})$, expressed as the Fourier transforms of the joint probability density functions $p_{1}(\widehat{u}, \widehat{w})$ and $p_{2}(\widehat{v}, \widehat{w})$, respectively, can be expressed as function of the moment and cumulant generating functions in which $m_{1, j k}=\overline{\widehat{u}^{j} \widehat{w}^{k}}$ and $m_{2, j k}=\overline{\widehat{v}^{j} \widetilde{w}^{k}}$ denote the moments of $(j+k)$ th order and $k_{1, j k}$ and $k_{2, j k}$ correspond to the cumulants of $(j+k)$ th order. Nakagawa and Nezu [16] expressed the conditionally sampled probability densities over the four quadrants of covariance events $\varepsilon_{1}$. The mathematical manipulations are completely described in [16]. The following equations are given:

$$
\begin{aligned}
& p_{i, 2}\left(\varepsilon_{i}\right)=p_{i N}\left(\varepsilon_{i}\right)+\varphi_{i}^{-}\left(\varepsilon_{i}\right) \\
& p_{i, 4}\left(\varepsilon_{i}\right)=p_{i N}\left(\varepsilon_{i}\right)-\varphi_{i}^{-}\left(\varepsilon_{i}\right) \quad\left(\varepsilon_{i}>0\right) \\
& p_{i, 1}\left(\varepsilon_{i}\right)=p_{i N}\left(\varepsilon_{i}\right)+\varphi_{i}^{+}\left(\varepsilon_{i}\right) \\
& p_{i, 3}\left(\varepsilon_{i}\right)=p_{i N}\left(\varepsilon_{i}\right)-\varphi_{i}^{+}\left(\varepsilon_{i}\right) \quad\left(\varepsilon_{i}<0\right)
\end{aligned}
$$

where the index $q$ in $p_{i, q}$ denotes the quadrant index in the $i$ th plane with planes 1 and 2 corresponding to the longitudinal and transverse planes, respectively. The probability density $p_{i N}\left(\varepsilon_{i}\right)$ is directly developed from the corresponding bivariate normal distribution. The nonconditionally sampled probability function of shear stress is $p_{i}\left(\varepsilon_{i}\right)=p_{i, 1}\left(\varepsilon_{i}\right)+p_{i, 2}\left(\varepsilon_{i}\right)+p_{i, 3}\left(\varepsilon_{i}\right)+$ $p_{i, 4}\left(\varepsilon_{i}\right)=2 p_{i, N}$ with $i=1,2$ and

$$
\left\{\begin{array}{l}
p_{i N}\left(\varepsilon_{i}\right)=\frac{r_{i}}{2 \pi} \exp \left(r_{i} t_{i}\right) \frac{K_{0}\left(\left|t_{i}\right|\right)}{\left(1-r_{i}^{2}\right)^{1 / 2}} \\
\varphi_{i}^{+}\left(\varepsilon_{i}\right)=\frac{r_{i}}{2 \pi} \exp \left(r_{i} t_{i}\right) K_{1 / 2}\left(\left|t_{i}\right|\right) \frac{\left|t_{i}\right|^{1 / 2}}{\left(1-r_{i}^{2}\right)} \\
\quad\left[\left(1+r_{i}\right)\left(\frac{S_{i}^{+}}{3}+D_{i}^{+}\right)\left|t_{i}\right|-\left(\frac{2-r_{i}}{3} S_{i}^{+}+D_{i}^{+}\right)\right] \\
\varphi_{i}^{-}\left(\varepsilon_{i}\right)=\frac{r_{i}}{2 \pi} \exp \left(r_{i} t_{i}\right) K_{1 / 2}\left(\left|t_{i}\right|\right) \frac{\left|t_{i}\right|^{1 / 2}}{\left(1+r_{i}^{2}\right)} \\
{\left[\left(1-r_{i}\right)\left(\frac{S_{i}^{-}}{3}+D_{i}^{-}\right)\left|t_{i}\right|-\left(\frac{2+r_{i}}{3} S_{i}^{-}+D_{i}^{-}\right)\right]} \\
t_{i}=\frac{r_{i} \varepsilon_{i}}{\left(1-r_{i}^{2}\right)} ; \\
D_{i}^{ \pm}=\frac{1}{2}\left(k_{i, 21} \pm k_{i}^{ \pm}=\frac{1}{2}\left(k_{i, 03} \pm k_{i, 30}\right)\right.
\end{array}\right.
$$

where $r_{i}$ are the corresponding correlation coefficients and $K_{0}(t)$ is the zeroth-order modified Bessel function of the second kind. From (1), we will calculate the first-order moment of each conditional probability density distribution as a function of the threshold levels $H_{i}$. Increasing the level of $H_{i}$ allows the selection of progressively stronger fractional covariance events. Their distribution over the different quadrants in the longitudinal and transverse planes can be investigated using the following expressions:

$$
\begin{gathered}
R S_{i, q}\left(H_{i}\right)=\int_{H_{i}}^{\infty} \varepsilon_{i} p_{i, q}\left(\varepsilon_{i}\right) d \varepsilon_{i} \quad H_{i} \geq 0 \\
i=1,2 \quad q=2,4 \\
R S_{i, q}\left(H_{i}\right)=\int_{-\infty}^{H_{i}} \varepsilon_{i} p_{i, q}\left(\varepsilon_{i}\right) d \varepsilon_{i} \quad H_{i} \leq 0 \\
i=1,2 \quad q=1,3 .
\end{gathered}
$$

This method is known as the $u-w$ quadrant threshold technique and is described in [14]. The parameters $R S_{i, q}$, evaluated from the probability densities, will be compared to those from experimental results in order to obtain information about the quadrant distribution of the relative covariances. The time fractions $T_{i, q}$ of these conditionally sampled events are estimated by

$$
\begin{gathered}
T_{i, q}\left(H_{i}\right)=\int_{H_{i}}^{\infty} p_{i, q}\left(\varepsilon_{i}\right) d \varepsilon_{i} \quad H_{i} \geq 0 \\
i=1,2 \quad q=2,4 \\
T_{i, q}\left(H_{i}\right)=\int_{-\infty}^{H_{i}} p_{i, q}\left(\varepsilon_{i}\right) d \varepsilon_{i} \quad H_{i} \leq 0 \\
i=1,2 \quad q=1,3 .
\end{gathered}
$$

Consequently, those parts of the events having relative shear stress values lower than the defined thresholds are called the "hole event" terms (Fig. 2). $R S_{i, \Sigma}$ and $T_{i, \Sigma}$ are given by

$$
\begin{aligned}
R S_{i, 5}\left(H_{i}\right) & =1-\sum_{q=1}^{4} R S_{i, q}\left(H_{i}\right) \\
T_{i, 5}\left(H_{i}\right) & =1-\sum_{q=1}^{4} T_{i, q}\left(H_{i}\right) .
\end{aligned}
$$

\section{B. Results and Discussion}

Fig. 3 presents the theoretical and experimental quadrant fractional contributions of the relative covariance term $\varepsilon_{1}$ calculated from (3) using the $u^{\prime} w^{\prime}$-quadrant technique for experiment C. A comparison of the results at four different depths confirms the ejection-sweep dominance in $\varepsilon_{1}$ contributions. Good agreement is seen between the theoretical and the experimental distributions for a threshold level $H_{1}<10$. Above that value, deviations between the theoretical and the experimental curves point to the limitation of the third-order model because the covariance generation process becomes more intermittent with increasing $H_{1}$. However, the value of $T_{1,5}$ in Fig. 3 indicates that less than $10 \%$ of all events still contribute in that case. 


\section{longitudinal section $\left(\varepsilon_{1}\right.$ events)}

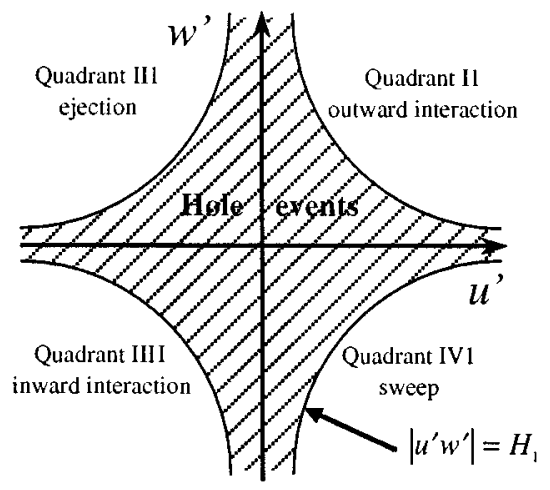

(a)

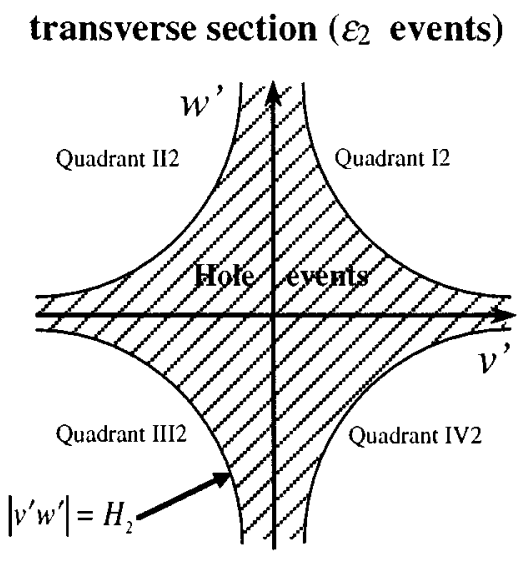

(b)

Fig. 2. Division of events in the (a) longitudinal and (b) transverse planes.

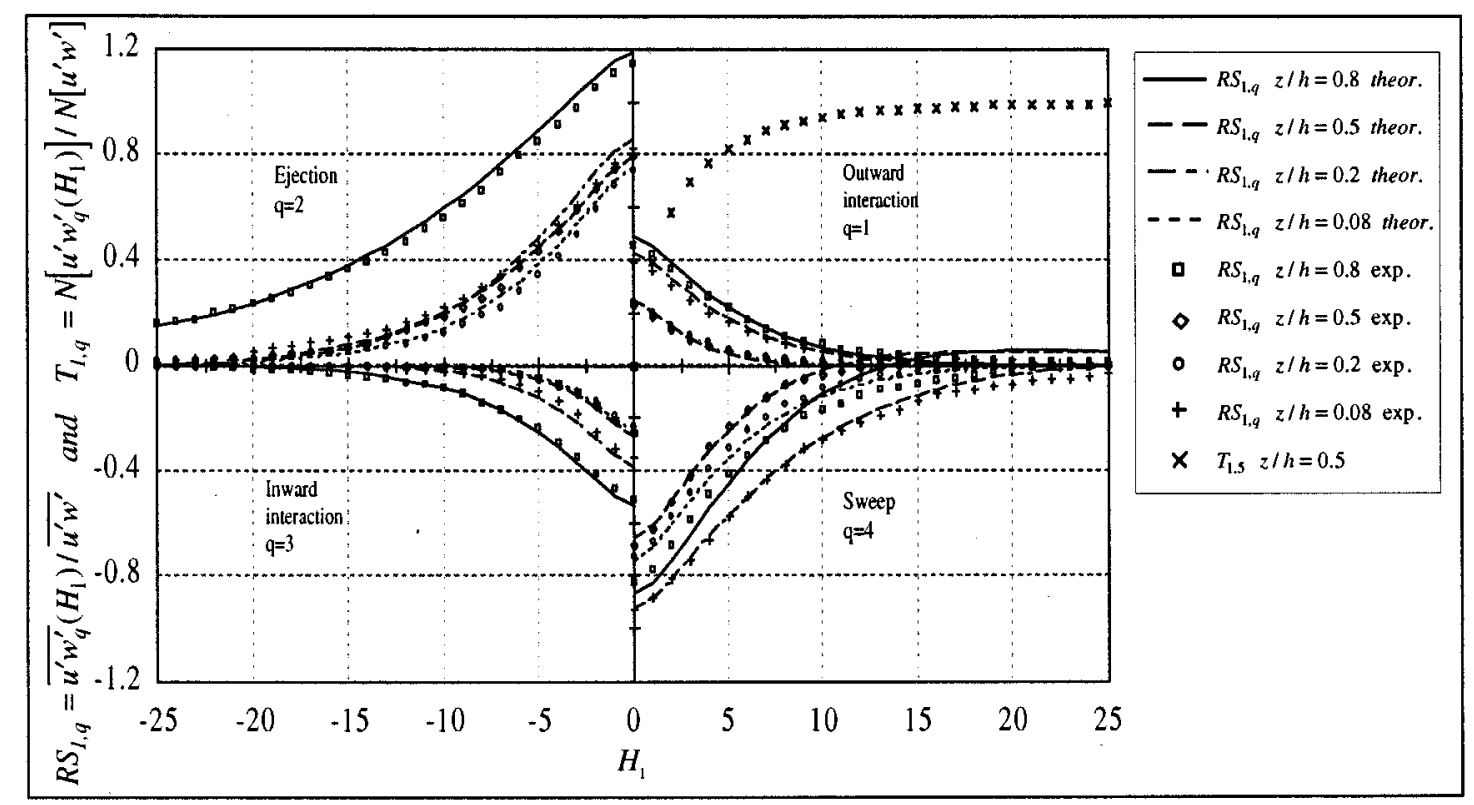

Fig. 3. Fractional contribution of relative covariance $\varepsilon_{1}$ versus threshold level $H_{1}$ at four flow depths and time fraction of hole event $T_{1,5}\left(z / h=0.5, H_{1}\right)$, for Experiment $\mathrm{C}$. The notation $N[x]$ represents the number of $x$ values.

In the outer region of the boundary layer $(z / h>0.2)$, ejections contribute more than sweeps. In the inner region, the sweep contribution increases and reaches the same level as the ejections. This was also observed by Grass [3] who stated that sweep events become larger in the vicinity of the bed. The contributions of inward (quadrant 3 ) and outward (quadrant 1) interactions are much lower, remaining nearly constant over the entire boundary layer depth.

The time fraction of the hole events $T_{1,5}$ at depth $z / h=$ 0.5 is also indicated in Fig. 3. The variation of $T_{1,5}$ with depth was found to be weak. A given $H_{1}$ value indicates the time fraction of those events which do not contribute to the $R S_{i, q}\left(H_{1}\right)$ level. For example, for $H_{1}=10$, the time fraction of the hole events is more than $90 \%$. Thus, less than $10 \%$ still contribute to the event generation. In that case (for $z / h=0.08$ ), $R S_{1,1}=0.05, R S_{1,3}=-0.02, R S_{1,4}=$ -0.28 , and $R S_{1,2}=0.2$, which characterize the turbulence generation process as a short-duration, high-magnitude event. This quantitative calculation confirms the qualitative observation in Fig. 1(a) and (b). Coherent structures such as ejections and sweeps are obviously related to the generation of important covariance events and, as a result, also act as the main turbulent energy producers. The results in Fig. 3 agree well with those previously described by several authors using hot film or LDA measurements.

In Fig. 4, the same quantities as in Fig. 3 are presented for the covariance events $\varepsilon_{2}$, in the transverse plane of the boundary layer. The curves fall off more slowly, stretching to much larger values of the threshold level $\mathrm{H}_{2}$, thus denoting larger $\varepsilon_{2}$ variations from their temporal mean and low mean covariance $\overline{v^{\prime} w^{\prime}}$. It is particularly striking that the $\varepsilon_{2}$ covariance generation is nearly equal for all quadrants, independent of the threshold level $\mathrm{H}_{2}$ and depth $z / h$. This quantitative calculation confirms the qualitative observation in Fig. 1(c) and (d). 


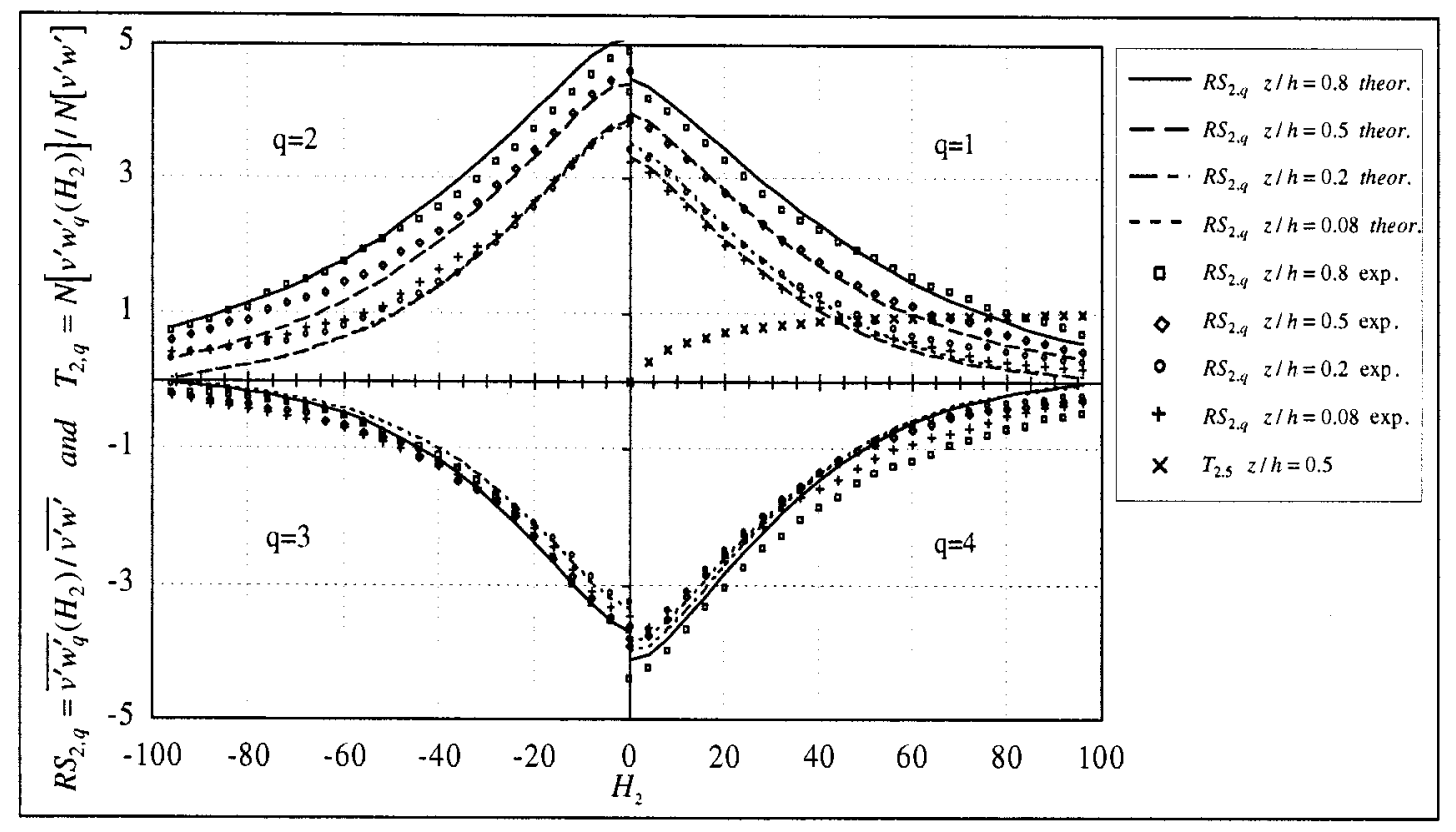

Fig. 4. Fractional contribution of relative covariance $\varepsilon_{2}$ versus threshold level $H_{2}$ at four flow depths and time fraction of hole event $T_{2,5}\left(z / h=0.5, H_{2}\right)$, for Experiment $\mathrm{C}$. The notation $N[x]$ represents the number of $x$ values.

In order to compare quantitatively the $R S_{i, q}$ and $T_{i, q}$ values of the different quadrants and planes, Fig. 5 represents their profiles over the depth for two different threshold levels $H_{1}=H_{2}$ and two bed roughness conditions (Experiments $\mathrm{A}$ and D). As indicated above, $R S_{1,2}$ and $R S_{1,4}$ contribute significantly to covariance events $\varepsilon_{1}, R S_{1,2}$ becoming larger than $R S_{1,4}$ toward the free surface independent of bed roughness. The effect of roughness is evident from an increase toward the bed of the ejection contribution in the rough bed case compared to the smooth bed case (at $z / h \approx 0.3$ ). In the wall region, the ejection contribution becomes lower than the sweep contribution at $z / h \approx 0.12$, whereas the ejection contribution remains greater than the sweep contribution in the smooth case. This tendency is independent of the parameter $H_{1}$.

We can observe from the $T_{i, q}$ profiles that even with very low time fraction of quadrant 2 and 4 events, their contribution remains important for high threshold values (for $H_{1}=8$ we find depth averaged values of $T_{1,2} \approx T_{1,4} \approx 0.04$ and $R S_{1,2}=0.4$ $R S_{1,4}=0.15$ ). The time fraction evolution with $H_{1}$ indicates a rapid decrease of $T_{1,1}$ and $T_{1,3}$ reaching a zero value at $H_{1}=4$ (not shown in Fig. 5) whereas $T_{1,2}$ and $T_{1,4}$ remain nearly equal except for $H_{1}=0$. Nakagawa and Nezu had previously given profiles for $\varepsilon_{1}$ events, but only for $H_{1}=0$ [16]. The presented results correspond well with theirs.

In Fig. 5, the fractional $\varepsilon_{2}$ covariance magnitudes $R S_{2, q}$ are normalized by $\sqrt{\overline{v^{\prime 2}} \overline{w^{\prime 2}}}$ because the low values of the term $\overline{v^{\prime} w^{\prime}}$ induce very high $R S_{2, q}$ values. As mentioned before, the contributions from the different quadrants are nearly the same and are independent of $z / h$ and $\mathrm{H}_{2}$. It can be seen that the time fractions only slightly vary with depth, remaining close to 0.25 for $H_{2}=0$ over the whole depth. From Figs. 4 and 5, we can confirm that no apparent quadrant related bursting process is observed for the covariance term $\varepsilon_{2}$ in the transverse flow plane.
The third-order cumulants (crossed and noncrossed) determine the repartition of the relative covariance contributions over the different quadrants. In (2), the terms $S_{1}^{-}, D_{1}^{-}, S_{2}^{+}$, and $S_{2}^{-}$ only depend on third-order cumulants. Therefore, a comparison of these moments for the different flow conditions gives information about the shear stress dynamics.

Fig. 6 shows all third-order moments needed to evaluate the factors and in (2) for experiment C. As previously observed by Nakagawa and Nezu [16] and Raupach [19], the $m_{1, j k}$ profiles are symmetrical over an extended range roughly corresponding to $0.2 \leq z / h \leq 0.8$. The relation between all four moments can be assumed to be linear [19]. Nakagawa and Nezu considered that $m_{1,30}$ and $m_{1,21}$ are the reflections of $m_{1,03}$ and $m_{1,12}$, respectively, on the zero axis but did not relate the crossed moments to the skewness factors [16]. In our study, 3-D velocity data are available from the ADVP measurements and the crossed third-order moment $m_{2,21}$ can be calculated whereas this term had to be approximated in the previous studies. In our case, a linear combination of these third-order moments yields

$$
\begin{aligned}
m_{1,30} & =-0.94 m_{1,03}=-1.78 m_{1,21}=1.43 m_{1,12} \\
& =-2.27 m_{2,21}, \quad \text { for } 0.25 \leq z / h \leq 0.75
\end{aligned}
$$

where the coefficients have been estimated by applying a least square fit to data included in the interval $0.25 \leq z / h \leq 0.75$. The mean correlation coefficients (averaged over the four experiments) are $R_{c_{1,03}}=0.82, R_{c_{1,12}}=0.81, R_{c_{1,21}}=0.87$, and $R_{c_{2,21}}=0.51$. In order to determine whether these numerical values of the coefficients linking the different third-order moments are constant and independent of Reynolds, Froude numbers, and bed roughness, (6) is presented for all experiments in Fig. 7. For $0.25 \leq z / h \leq 0.75$, the values of the different coefficients in (6) are found to be nearly equal for all investigated 


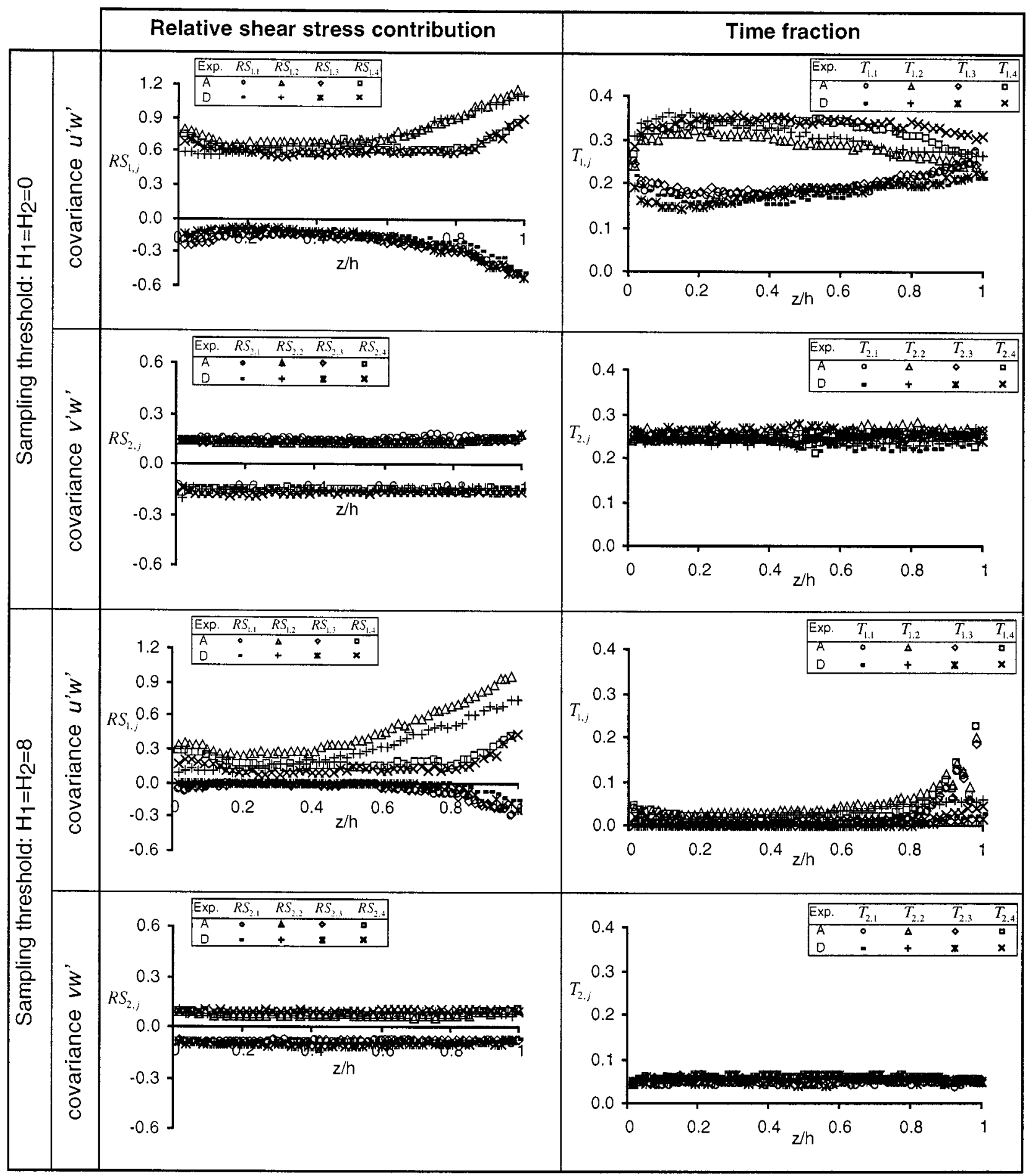

Fig. 5. Comparison of fractional covariance and time fractions of $\varepsilon_{1}$ and $\varepsilon_{2}$ versus flow depth at two $H_{1}=H_{2}$ threshold levels for experiment A (smooth) and experiment D (rough bed).

flow conditions. Slight deviations are observed in the wall and free surface flow regions.

\section{WALl SIMILARITY IN TURBULENT BOUNDARY LAYERS}

The wall similarity concept in turbulent boundary layer flow under uniform flow conditions is based on the existence of an extended region in depth where the turbulent energy production and dissipation terms are in equilibrium and the diffusion terms are negligible. The two diffusion terms (fluctuating pressure and turbulent kinetic energy diffusion) not only compensate each other but are nearly equal to zero [17]. Therefore, the similarity concept implies that, in uniform flows with high Reynolds numbers, a similar equilibrium flow region exists, independent of the flow conditions and bed roughness conditions. This con- 


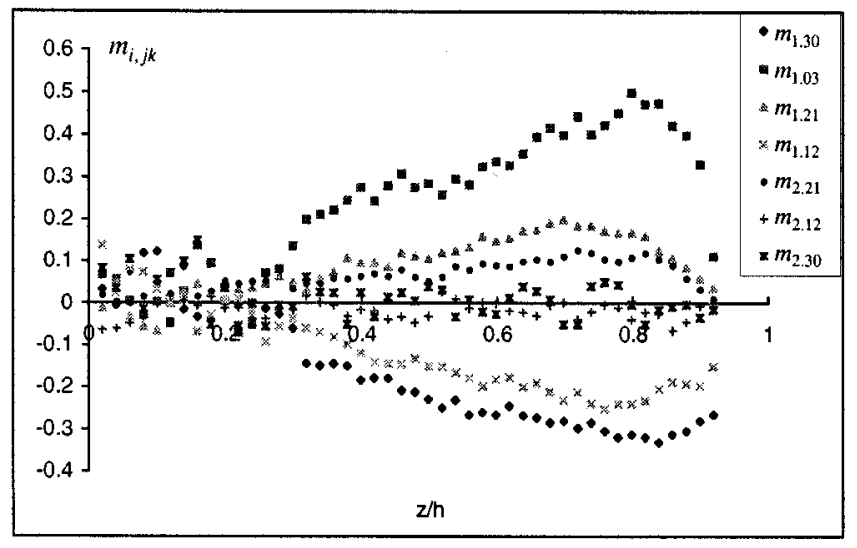

Fig. 6. Representation of third-order moments (experiment C).

cept can be described by the turbulent energy budget equation:

$$
\begin{aligned}
& \underbrace{\frac{\partial}{\partial t} \frac{\overline{q^{2}}}{2}+U_{k} \frac{\partial}{\partial x_{k}} \frac{\overline{q^{2}}}{2}}_{\text {TRANSPORT }}+\underbrace{\overline{u_{i}^{\prime} u_{k}^{\prime}} \frac{\partial U_{i}}{\partial x_{k}}}_{\text {PRODUCTION }} \\
& =\underbrace{-\frac{\partial}{\partial x_{k}} \overline{\left(\frac{p^{\prime}}{\rho}+\frac{q^{2}}{2}\right) u_{k}^{\prime}}}_{\text {TURBULENT DIFFUSTON }}+\underbrace{v \frac{\partial^{2}}{\left(\partial x_{l}\right)^{2}} \frac{\overline{q^{2}}}{2}}_{\text {VISCOUS DIFFUSION }} \\
& -\underbrace{\overline{v \frac{\partial u_{i}^{\prime}}{\partial x_{l}} \frac{\partial u_{i}^{\prime}}{\partial x_{l}}}}_{\text {DISSIPATION }}
\end{aligned}
$$

in which Einstein's notation convention is employed (i.e., $q^{2} / 2=u_{i}^{\prime 2} / 2$ is the turbulent kinetic energy). Fig. 8 shows the normalized shear stress profiles for all investigated experiments. The observed linear variations over the relative flow depth confirm that the flow is uniform and in the mean two-dimensional at high Reynolds number (see Table I). Hence, the last equation yields

$$
\begin{aligned}
& \underbrace{-\overline{u^{\prime} w^{\prime}} \frac{\partial \bar{u}}{\partial z}}_{\text {PRODUCTION }}= \underbrace{\frac{\partial}{\partial z}\left[\frac{1}{2} \overline{\left(u^{\prime 2}+v^{\prime 2}+w^{\prime 2}\right) w^{\prime}}+\overline{p^{\prime} w^{\prime}}\right]}_{\text {TURBULENT DIFFUSION }} \\
&+\underbrace{\varepsilon}_{\text {DISSIPATION }} \cdot
\end{aligned}
$$

The equilibrium concept is expressed as follows:

with

$$
-\overline{u^{\prime} w^{\prime}} \frac{\partial \bar{u}}{\partial z} \cong \varepsilon
$$

$$
\frac{1}{2} \overline{\left(u^{\prime 2}+v^{\prime 2}+w^{\prime 2}\right) w^{\prime}} \cong \text { const. for } 0.15 \leq z / h \leq 0.6 .
$$

The relation of the third-order moments, discussed in Section $\mathrm{V}$, to the normalized vertical flux of turbulent kinetic energy is given by (the normalization variable will be discussed later)

$$
\begin{aligned}
F_{k} & =\frac{1}{2 u_{*}^{3}} \overline{\left(u^{\prime 2}+v^{\prime 2}+w^{\prime 2}\right) w^{\prime}} \\
& =\frac{\sqrt{\overline{w^{\prime 2}}}}{2 u_{*}^{3}}\left(\overline{u^{\prime 2}} m_{1,21}+\overline{v^{\prime 2}} m_{2,21}+\overline{w^{\prime 2}} m_{1,03}\right) .
\end{aligned}
$$

This equation indicates a direct connection between the turbulent energy flux and the dynamics of the shear stress events through the third order moments. As shown previously, these statistical parameters determine the distribution of the relative covariance terms over the different quadrants. Furthermore, the statistical analysis has shown that the relation between the different third order moments is linear over a flow region exceeding the intermediate flow region [see (2)]. This is represented by the following expression:

$$
\left\{\begin{array}{l}
m_{1,30}=c_{1,03} m_{1,03} \\
m_{1,30}=c_{1,21} m_{1,21} \\
m_{1,30}=c_{1,12} m_{1,12} \\
m_{1,30}=c_{2,21} m_{2,21,} \quad \text { for } 0.25 \leq z / h \leq 0.75 .
\end{array}\right.
$$

If the relations between the different turbulence intensities are used, i.e.,

$$
\sqrt{\frac{\overline{v^{\prime 2}}}{\overline{u^{\prime 2}}}}=C_{1} \quad \sqrt{\frac{\overline{w^{\prime 2}}}{\overline{u^{\prime 2}}}}=C_{2}
$$

then (10) can be rewritten as a function of only one third-order moment as follows:

$$
F_{k}=S \frac{\overline{u^{\prime 3}}}{u_{*}^{3}}
$$

with

$$
\begin{gathered}
S=\frac{C_{2}}{2}\left(\frac{1}{c_{1,21}}+\frac{C_{1}^{2}}{c_{2,21}}+\frac{C_{2}^{2}}{c_{1,03}}\right), \\
\text { for } 0.25 \leq z / h \leq 0.75
\end{gathered}
$$

Nakagawa and Nezu [16] derived the following expression for (10):

$$
F_{k} \cong \frac{1}{2}\left(\frac{\sigma_{w}}{\sigma_{u}}\right)\left(\frac{\sigma_{u}}{u_{*}}\right)^{3}\left[m_{1,21}+2\left(\frac{\sigma_{w}}{\sigma_{u}}\right)^{2} m_{1,03}\right]
$$

with

$$
\sigma_{i}=\sqrt{\overline{{u_{i}^{\prime 2}}_{i}}}
$$

by considering that $m_{1,30}$ and $m_{1,21}$ are the reflections of $m_{1,03}$ and $m_{1,12}$, respectively, on the zero axis. They did not relate the crossed moments to the skewness factors. Since they did not have 3-D measurements, they approximated the crossed third-order moment $m_{2,21}$ in (10).

We evaluated (10), (13), and (14) using our data. The results are plotted in Fig. 9. The three curves coincide except in the inner flow region. Some of the differences between them might be due to the use of (12) which are only valid over the whole 


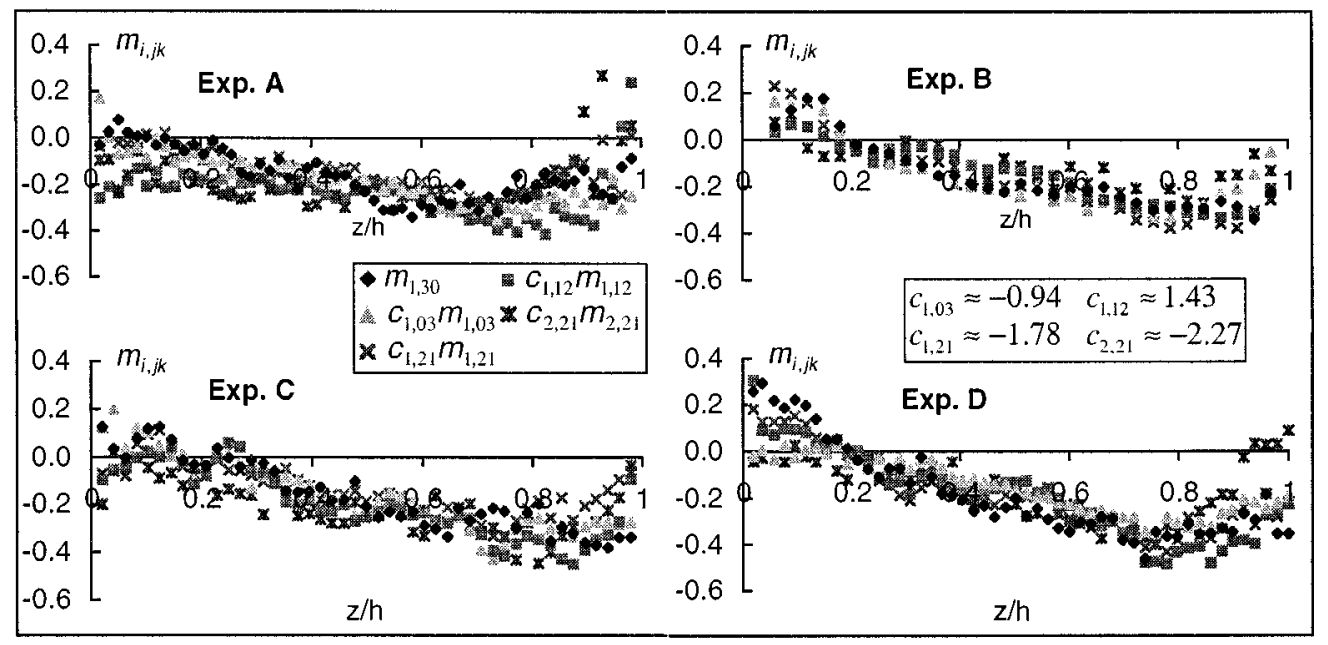

Fig. 7. Observed constant relationship between third-order moments for experiments A-D.



Fig. 8. Shear stress profiles for all experiments.

depth if the bed is smooth. In the presence of roughness elements, the turbulence intensity profiles deviate considerably from (12) in the wall region [17].

\section{BotTOM SHEAR VELOCITY IN TURbULENT BOUNDARY LAYERS}

The statistical analysis (Section V, Fig. 7) has shown that the factors relating the third-order moments are independent of Reynolds number, Froude number, and bed roughness over a depth range corresponding to roughly $0.25 \leq z / h \leq 0.75$. Therefore, when properly normalized, the vertical flux of turbulent kinetic energy should be constant over that range of depths. We normalized it by the cube of the bed friction velocity, which takes into account the bed roughness effect through the energy slope and boundary layer depth. Fig. 10 presents the normalized flux for all our experiments. In all cases, we found a nearly constant flux for $0.25 \leq z / h \leq 0.75$ with values varying between 0.28 to 0.35 . In a recent study using point measurements, López and Garcia [13] found a value of 0.33 over a smaller range of depths but a wider range of bottom roughness. They concluded that a constant value of 0.3 may be universal, which is in good agreement with the present results.



Fig. 9. Validation of a simplified relation of $F_{k}$ (data of experiment C).

The observed similarity provides another method for determining the mean bed friction velocity, since (13) requires knowledge of only one instantaneous velocity component [in comparison with (10) or (14) in which the crossed third-order moments are estimated from 3-D or 2-D velocities measurements, respectively]. Taking $C_{1}=0.75$ and $C_{2}=0.35$, we found $S \cong-0.35$ from our data. Using the value of $F_{k}$ from Fig. 10 in (13), the mean bottom friction velocity can then be determined. Due to the one-third power relationship, this method of calculation is not very sensitive to even large errors in the determination of the energy flux.

Difficulties and advantages of different methods for the determination of the mean bottom friction velocity are discussed in [13]. In field studies, they suffer either from the lack of knowledge of the precise location of the bed reference level, or the restricted range of validity of the underlying assumptions. In the present study, we found the similarity concept to be valid over a wide depth range for bottom roughnesses which are typical of oceanic boundary layers. The precision of the determination of the measurement position within that range, and thus the bed reference level, is therefore not critical. Furthermore, this range is well above the bed where the gradient of the mean velocity is weak. Measurement techniques with larger measurement volumes may therefore be applied in this case. 


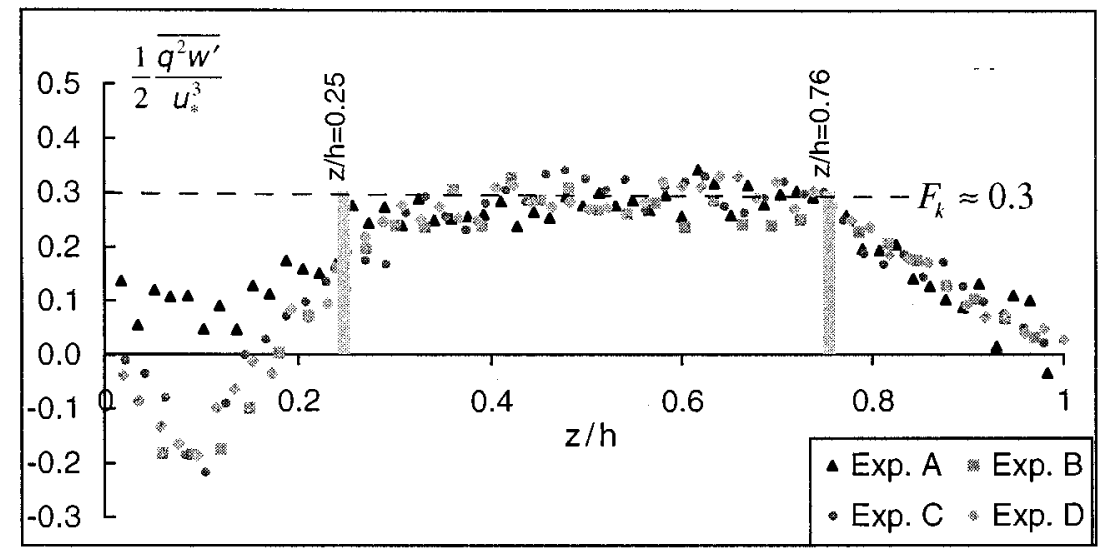

Fig. 10. Normalized flux of turbulent kinetic energy (for experiments A-D).

Based on our study of noise sources in 3-D ADVP turbulence measurements, discussed in [6], we found noise characteristics which indicate that, even for third-order moment estimations from 3-D ADVP data, the SNR remains high as long as errors related to acquisition time and bandwidth can be neglected.

\section{SUMMARY AND CONCLUSIONS}

Velocity and shear stress fields obtained by high-resolution 3-D Doppler velocity profiling in free-surface boundary layer flows over smooth and rough beds show coherent structures extending over a large portion of the boundary layer depth that are approximately independent of bottom roughness. Structural features such as ejections, sweeps, upwellings, downdrafts, vorticity patterns, and spiral eddies can be identified when compared to tracer measurements found in [4]. Recent studies [9] have shown that these coherent structures can be linked to surface boils. Coherent structures therefore play an important role in the dynamics of air-water transfer processes.

Good agreement is found between these qualitative observations and a statistical analysis of our measurements, which clearly exhibit the well-known quadrant distribution in the longitudinal plane. The effect of bottom roughness in changing the balance between ejections and sweep is important only below $0.2 \mathrm{~h}$. The quadrant decomposition of the transverse covariance term, which has not been estimated before, is found to be almost uniform over the four quadrants. This confirms our initial qualitative observations from figures. It also indicates that the organization of the flow in ejection and sweep events is essentially limited to the vertical plane defined by the mean flow direction.

The approximation of the conditional probability densities of the covariance terms $\varepsilon_{1}=u^{\prime} w^{\prime} \overline{u^{\prime} w^{\prime}}$ and $\varepsilon_{2}=v^{\prime} w^{\prime} / \overline{v^{\prime} w^{\prime}}$ by a third-order Gram-Charlier expansion appears to be sufficient for thresholds $H_{i}<10$. Above that value, the third-order cumulant discard model deviates from the observations. This suggests that the processes of shear stress generation and turbulent energy production in the boundary layer are highly intermittent.

Based on the our measurements of the statistical properties of the covariance terms for different flow conditions, we have validated the wall similarity concept. We found a constant value of 0.3 for the normalized vertical flux of turbulent kinetic energy over a range of depths exceeding the equilibrium flow region, independent of the flow conditions investigated here. This agrees with the value found by López and Garcia [13] for a different range of bottom roughness.

A different approximation for the turbulent kinetic energy flux, valid in the observed equilibrium flow depth range, is developed in (13). It depends on the longitudinal fluctuating velocity component only, which considerably simplifies the theoretical expression of the turbulent kinetic energy flux.

The existence of wall similarity in highly turbulent boundary layers offers a new method of determining the mean bed friction velocity. This method is better adapted to field measurements than previous methods because the measurements can be taken at a single level far from the bed over an extended depth range where the gradient of the mean flow profile is weak. In particular, this avoids the difficulty of needing to know the precise bed reference level, a difficulty that has introduced large errors in classical calculations based on profile measurements. Here we have shown that this concept is valid at the center of shallow, uniform, open-channel flows at high Reynolds number where the boundary layer is 2-D in the mean. Under oceanic conditions, effects such as rotation and stratification may cause modifications. Recently, we have studied the structure and the dynamics of secondary currents in uniform flow over rough beds by taking closely spaced 3-D ADVP profiles across a channel cross section. It was demonstrated that in the mean the wall similarity concepts still holds despite the presence of secondary currents. In order to determine the range of validity of the method presented here, field investigations are needed.

This study has demonstrated the potential of a high-resolution 3-D acoustic Doppler velocity profiler in turbulent boundary layer flow. It is ideally suited for such studies because it can profile the total depth in one sweep. This is particularly important in boundary layer field studies where flow conditions often change rapidly and point-by-point measurements will fail to provide the details desired to understand the high-frequency dynamics of the boundary layer. As we have shown recently, this approach can be extended to measure sediment flux [22], which will make it possible to carry out studies similar to the one presented here in sediment-laden flows [7]. New insight into sediment transport dynamics can be expected from measurements with this instrument. 


\section{ACKNOWLEDGMENT}

The authors would like to thank the anonymous reviewers for constructive comments and suggestions which helped to improve and clarify the presentation.

\section{REFERENCES}

[1] R. A. Antonia and J. D. Atkinson, "High-order moments of Reynolds shear stress fluctuations in a turbulent boundary layer," J. Fluid Mech. vol. 58, no. 3, pp. 581-593, 1973.

[2] W. H. Graf and M. Altinakar, Fluvial Hydraulics. New York, NY: Wiley, 1998, p. 681

[3] A. J. Grass, "Structural features of turbulent flow over smooth and rough boundaries," J. Fluid Mech., vol. 50, no. 2, pp. 233-255, 1971.

[4] A. J. Grass, R. J. Stuart, and M. Mansour-Tehrani, "Common vortical structure of turbulent flows over smooth and rough boundaries," AIAA J., vol. 31, no. 5, pp. 837-847, 1993.

[5] D. Hurther and U. Lemmin, "A constant beamwidth transducer for 3-D acoustic Doppler profile measurements in open channel flows," Meas. Sci. Technol., vol. 9, no. 10, pp. 1706-1714, 1998.

[6] — "A correction method for turbulence measurements with a 3-D Acoustic Doppler Velocity Profiler,” J. Atmos. Oceanic Technol, 1999, to be published.

[7] _ - "Particle transport capacity of coherent structures in suspensions flows," Experiments in Fluids, 2000, submitted for publication.

[8] S. J. Kline, W.C. Reynolds, F. A. Schraub, and P. W. Rundstadler, "The structure of turbulent boundary layers," J. Fluid Mech., vol. 30, pp. 741-773, 1967.

[9] S. Kumar, R. Gupta, and S. Banerjee, "An experimental investigation of the characteristics of the free surface turbulence in channel flow," Phys. Fluids, vol. 10, no. 2, pp. 437-456, 1998

[10] R. Lhermitte and R. Serafin, "Pulse-to-pulse coherent Doppler sonar signal processing techniques," J. Atmos. Oceanic Technol., vol. 1, pp. 293-308, 1984.

[11] R. Lhermitte and U. Lemmin, "Open-channel flow and turbulence measurement by high-resolution Doppler sonar," J. Atmos. Oceanic Technol., vol. 11, pp. 1295-1308, 1994.

[12] U. Lemmin, D. Hurther, and C. Shen, "A high resolution constant beam width pulse-to-pulse coherent 3-D acoustic Doppler profiling current meter for use in laboratory and environmental studies," in Proc. IEEE Sixth Conf. Current Measurements, San Diego, CA, 1999, pp. 216-220.

[13] F. López and M. H. Garcia, "Wall similarity in turbulent open-channel flow," J. Eng. Mech., vol. 125, no. 7, pp. 789-796, 1999.

[14] S. S. Lu and W. W. Willmarth, "Measurements of the structure of the Reynolds stress in a turbulent boundary layer," J. Fluid Mech., vol. 60, pp. 481-511, 1973
[15] Y. Nagano and M. Tagawa, "Coherent motions and heat transfer in a wall turbulent shear flow," J. Fluid Mech., vol. 305, pp. 127-157, 1995.

[16] H. Nakagawa and I. Nezu, "Prediction of the contribution to the Reynolds stress from bursting events in open-channel flows," J. Fluid Mech., vol. 80, no. 1, pp. 99-128, 1977.

[17] I. Nezu and H. Nakagawa, "Turbulence in open channel flows," in AIHR Monograph Series: Balkema, 1993, p. 281.

[18] R. Pinkel, "Observations of nonlinear motion in the open sea using a range-gated Doppler sonar," J. Phys. Oceanogr., vol. 9, pp. 675-680, 1979.

[19] M. R. Raupach, "Conditional statistics of Reynolds stress in rough wall and smooth wall turbulent boundary layers," J. Fluid Mech., vol. 108 , pp. 363-382, 1981

[20] S. K. Robinson, S. J. Kline, and P. R. Spalart, "Quasicoherent structures in the turbulent boundary layer: Part II. Verification and new information from a numerically simulated flat-plate layer," in Near-wall turbulence, Zoran Zaric Memorial Conference, Dubrovnik, Croatia, 1988, pp. 218-247.

[21] T. Rolland and U. Lemmin, "A two-component acoustic velocity profiler for use in turbulent open-channel flow," J. Hydr. Res., vol. 33, no. 4, pp. 545-561, 1997.

[22] C. Shen and U. Lemmin, "Ultrasonic scattering in highly turbulent clear water flow," Ultrasonics, vol. 35, pp. 57-64, 1997.

[23] — , "Application of an acoustic particle flux profiler in particle-laden, open-channel flow," J. Hydr. Res., vol. 37, no. 3, pp. 407-419, 1999. 